\title{
Acadêmicos de enfermagem do interior do Amazonas e o primeiro contato com uma unidade de terapia intensiva: relato de experiência
}

\author{
Nursing students from the interior of Amazonas and the first contact with an intensive care \\ unit: experience report
}
Estudiantes de enfermería del interior de Amazonas y el primer contacto con una unidad de cuidados intensivos: informe de experiencia

Stefany Alencar de Oliveira ${ }^{1 *}$, Karllen Adriane Bezerra da Silva ${ }^{1}$, Dhienifã Brena Marinho de Souza ${ }^{1}$, Stéfany Caetano Corrêa ${ }^{1}$, Deyvylan Araujo Reis ${ }^{1}$.

\begin{abstract}
RESUMO
Objetivo: Descrever a experiência vivida por acadêmicos do curso de enfermagem durante uma visita técnica a Unidade de Terapia Intensiva (UTI) de um Hospital de Alta Complexidade. Relato de Experiência: A unidade de terapia intensiva de um hospital de média e alta complexidade é caracterizada por ser um setor com sistema de monitorização contínua, ou seja, ininterrupto, que admite pacientes potencialmente graves com uma determinada patologia, podendo também ser advindos de um pós-operatório. A visita foi de grande importância, pois ajudou a atrelar a teoria com a prática, servindo para ampliar os conhecimentos dos alunos sobre a Unidade de Terapia Intensiva e o trabalho do enfermeiro intensivista, essa visita também oportunizou aos discente compreender a estrutura física e funcional, facilitando o entendimento acerca dos assuntos relacionados à unidade. Além disso, foi apresentado técnicas e protocolos empregados no setor para o manejo dos pacientes durante assistência de enfermagem. Considerações Finais: Portanto, a inserção nesse ambiente proporcionou maior compreensão acerca da complexidade de uma UTI, sendo uma experiência enriquecedora e essencial, contribuindo com a qualificação e crescimento profissional dos acadêmicos, uma vez que foi o primeiro contato o setor, produzindo, conhecimento e gerando novas experiências.
\end{abstract}

Palavras-chave: Conhecimento, Enfermagem, Unidade de terapia intensiva.

\begin{abstract}
Objective: Describe the experience of nursing students during a technical visit to the Intensive Care Unit (ICU) of a High Complexity Hospital. Experience report: The intensive care unit of a medium and high complexity hospital is characterized by being a sector with a continuous monitoring system, that is, uninterrupted, which admits potentially serious patients with a certain pathology, and may also come from a postoperative. The visit was of great importance, as it helped to link theory with practice, serving to expand students' knowledge about the Intensive Care Unit and the work of the intensive care nurse, this visit also gave students an opportunity to understand the physical and functional structure, facilitating the understanding about the issues related to the unit. In addition, techniques and protocols used in the sector for the management of patients during nursing care were presented. Final considerations: Therefore, the insertion in this environment provided a greater understanding of the complexity of an ICU, being an experience enriching and essential, contributing to the qualification and professional growth of academics, since it was the first contact with the sector, producing, knowledge and generating new experiences.
\end{abstract}

Key words: Knowledge, Nursing, Intensive care unit.

1Universidade Federal do Amazonas, Coari - Amazonas. *E-mail: Stefany152011@gmail.com 


\section{RESUMEN}

Objetivo: Describa la experiencia de los estudiantes de enfermería durante una visita técnica a la Unidad de Cuidados Intensivos (UCI) de un Hospital de Alta Complejidad. Informe de experiencia: la unidad de cuidados intensivos de un hospital de mediana y alta complejidad se caracteriza por ser un sector con un sistema de monitoreo continuo, es decir, ininterrumpido, que admite pacientes potencialmente graves con una determinada patología, y también puede provenir de un postoperatorio La visita fue de gran importancia, ya que ayudó a vincular la teoría con la práctica, sirviendo para ampliar el conocimiento de los estudiantes sobre la Unidad de Cuidados Intensivos y el trabajo de la enfermera de cuidados intensivos, esta visita también les brindó la oportunidad de comprender la estructura física y funcional, facilitando la comprensión de los problemas relacionados con la unidad. Además, se presentaron técnicas y protocolos utilizados en el sector para el manejo de pacientes durante la atención de enfermería. Consideraciones finales: Entonces La inserción en este entorno proporcionó una mayor comprensión de la complejidad de una UCI, siendo una experiencia enriquecedor y esencial, contribuyendo a la calificación y al crecimiento profesional de los académicos, ya que fue el primer contacto en el sector, produciendo, conociendo y generando nuevas experiencias.

Palabras clave: Unidad de conocimiento, Enfermería, Cuidados intensivos.

\section{INTRODUÇÃO}

De acordo com Ouchi JD, et al. (2018), durante a guerra da Crimeia, que ocorreu no ano de 1954, a precursora da enfermagem Florence Nightingale realizou, a partir da observação de que a alta taxa de mortalidade entre os soldados estava relacionado ao ambiente insalubre, a classificação dos feridos com base na complexidade dos ferimentos, em que uma atenção maior fosse direcionado aos casos mais graves, de modo que esses pacientes não ficassem desassistidos em nenhum momento, ou seja, com a verificação dos parâmetros vitais continuamente pela equipe de enfermagem. Tomando essas ações de Florence como referência foi concebida a Unidade de Terapia Intensiva (UTI).

Dentro do hospital a UTI é conhecida por ser um setor angustiante e apreensivo, criado para suprir a necessidade de atendimento a pacientes que precisam de assistência mais especializada e complexa, contando com recursos humanos e materiais competentes e altamente capacitados. A equipe da UTI é formada por profissionais altamente capacitados para oferecer atendimento contínuo e ininterrupto, utilizando os mais sofisticados equipamentos tecnológicos necessários ao diagnóstico, monitorização e terapia (SILVA GFD, et al., 2007; FERNANDES HS, et al., 2011).

A UTI é um local que contém uma alta concentração de tecnologias e diversas especializações, conta com uma equipe de saúde multiprofissional, formada por diversas profissões como enfermeiro, fisioterapeutas, médicos, nutricionistas entre outros, profissionais esses detentores de grande aporte de conhecimento, destreza e agilidade para a realização de procedimentos (BRASIL, 2005).

$\mathrm{Na}$ UTI o profissional enfermeiro exerce diversas atividades dentre as quais se encontram funções administrativas e assistenciais, entre elas: provisão de recursos humanos e materiais, executar, planejar e avaliar a assistência prestada, supervisionar os cuidados, bem como realizar educações em saúde aos familiares e pacientes. Sendo assim, a equipe de enfermagem tem como sua principal preocupação, o homem; tem como referencial a prática de cuidar, onde o objetivo fundamental é melhoria da qualidade de vida (MORTON PG, et al., 2007; SILVA GFD, et al., 2007).

A oportunidade de realizar as visitas técnicas possibilita aos acadêmicos alinhar os conhecimentos teóricos cientifico na prática, contribuindo, assim, no processo de construção de um profissional capaz de sugerir mudanças e desfechos para as dificuldades vivenciados, bem como melhorias na qualidade da assistência prestada ao paciente As visitas técnicas podem ser entendidas como um instrumento essencial ao processo de ensino-aprendizagem na graduação, que colabora de forma expressiva na formação acadêmica (LEITE C, et al., 2019). 
Dias EP, et al. (2014) diz que o acadêmico de enfermagem ao depararem pela primeira vez com um ambiente desconhecido, vivencia diversos sentimentos que variam entre a ansiedade, o medo e insegurança, estimulando diferentes expectativas. Sendo a UTI um setor com várias especialidades e apresentando um vasto campo de conhecimento, é mais recomendado para realização de visitas técnicas e estágio curricular, onde os estudantes podem ser capacitados para atender e prestar assistência de enfermagem a pacientes com disfunções orgânicas graves (CASATE JC e CORRÊA AK, 2006).

De acordo com Burgatti JC, et al. (2013), a Unidade de Terapia Intensiva estimula o desenvolvimento do pensamento crítico, reflexivo aos futuros profissionais de enfermagem, pois é um ambiente voltado a assistência e cuidados de pacientes que necessitam de assistência intensiva, complexa e integral para suporte e manutenção de suas funções vitais.

Diante do exposto a visita técnica realizada na UTI trouxe aos alunos a oportunidade de visualizar toda a área física do setor, materiais e equipamentos que são essenciais para o funcionamento desta unidade, Além do mais, foi possível observar o funcionamento dos aparelhos que estavam em uso e a rotina de trabalho da equipe multiprofissional que atende neste recinto. Com isso o presente estudo tem como objetivo descrever a experiência vivida por quatro acadêmicas do curso de enfermagem durante a visita técnica na UTI de um hospital de alta complexidade em Manaus-AM.

\section{RELATO DE EXPERIÊNCIA}

Trata-se de um estudo descritivo, do tipo relato de experiência, sobre a vivência de quatro acadêmicas do 9 o período de enfermagem de uma Universidade pública, localizado no interior do Amazonas, acerca da visita técnica, que foi realizada no mês de novembro de 2019 na UTI de um Hospital Universitário que recebe estudantes de vários cursos do ensino superior da região. O hospital onde foi realizada a visita técnica atende casos de média e alta complexidade no estado do Amazonas/Brasil. Neste local as acadêmicas puderam conhecer os materiais e equipamentos que compões a UTI do referido hospital, e na oportunidade aprenderam a manusear tais equipamentos, também tiveram a oportunidade de apreciar as funções administrativas e assistenciais que são exercidas pelos enfermeiros dentro desta unidade.

A UTI deste Hospital conta com uma estrutura física e equipamentos sofisticados, leitos modernos, que possuem bomba de infusão, ventilador mecânico, monitor multiparâmetro, entre outros equipamentos. Atualmente atua com 8 (oito) leitos ativos e conta com uma equipe de multiprofissionais e multidisciplinar sendo estes atuantes: enfermeiros administrativos e assistencial, técnicos de enfermagem, médicos especialistas e residentes de várias áreas da saúde, cujo o regime de plantão é de 30 horas semanais distribuídos em escalas de 12 (doze) e 6 (seis) horas por equipe de plantão, tudo para melhor atender e proporcionar conforto aos pacientes.

Os dois dias de visita técnica realizada na UTI do referido hospital, ocorreu com o intuito de promover aos discentes um maior conhecimento sobre as estruturas física e funcional, assim como, os cuidados de enfermagem empregados, visando também capacitar os alunos acerca da assistência de enfermagem ao paciente crítico; assim como, a UTI de outros hospitais essa unidade presta atendimento qualificado e sistematizado com monitorização contínua de seus clientes.

No primeiro dia de visita iniciamos fazendo o reconhecimento do setor, em que conhecemos a rotina da equipe de enfermagem, o funcionamento de cada departamento, lugares que ficam os materiais, todos os processos de assistência prestada e cada equipe que compõem esta unidade.

Nesse momento foi possível visualizar tanto a estrutura local quanto do cliente à beira do leito e as tecnologias da qual faz uso, como respiradores, monitores, entre outros. A Enfermeira que nos acompanhou durante a visita esclareceu todas as dúvidas, apresentou a parte técnica e clínica da UTI, desde equipamentos, materiais, prontuários dos pacientes, os registros de cada especialidade clínica, forma de admissão na unidade, preparação de medicação e alimentação enteral/parenteral e o estado de saúde de cada paciente que estava internado. 
Outro ponto muito relevante observado durante a visita foi à limpeza, pois se trata de um ambiente em que os pacientes apresentam quadros extremamente críticos, com o sistema imunológico comprometido, e alguns em restrição de contato e, portanto, mais suscetíveis às infecções, em vista disso entende-se a importância das medidas de assepsia e antissepsia adotadas no ambiente e durante os procedimentos respectivamente, além dos cuidados profiláticos para a realização das visitas por familiares. Esta atividade inicial foi muito relevante, pois não tínhamos nenhuma experiência quanto a uma Unidade Terapia Intensiva.

Também foi notado que para resguardar a saúde e a segurança, tanto dos clientes quanto dos profissionais, são indispensavelmente utilizados os Equipamentos de Proteção Individual (EPI) e os Protocolos de Segurança do Paciente, sendo este último como base de orientação para ofertar uma assistência adequada e de qualidade visando evita reações adversas na prestação de serviços de saúde. No decorrer das vivências em UTI, se observou que existem materiais de uso individual e coletivo, bem como equipamentos prontamente disponíveis para a prestação de serviços de saúde.

Assim, na condição de unidade por pacientes críticos que requerem equipamentos sofisticados, o número destes supre a total necessidade destes pacientes por se tratar de uma unidade nova e bem equipada, o que gera regulações no processo de trabalho para dar conta da demanda. Neste primeiro momento a experiência vivenciada mostrou que é possível desenvolver competências e habilidades no ensino clínico prático em alta complexidade da graduação a partir das atividades de visitas técnicas comparando a teoria com a prática.

No segundo dia as atividades desenvolvidas foram basicamente a realização e continuidade das atividades anteriormente mencionadas. Na unidade, percebe-se que a Enfermagem atua com liderança, e fomos orientados enfaticamente acerca dos planejamentos e atividades desenvolvidas neste âmbito, através de planos, metas e objetivos a serem alcançados anualmente com intuito de aprimorar a qualidade da assistência e assim como o trabalho dos profissionais.

O que nos causou maior impacto durante a visita foi que devido o tempo de permanência na UTI, que contribuiu para o desenvolvimento de transtornos como estresse e ansiedade, tanto do paciente quanto do profissional. No que tange a atuação do enfermeiro como coordenador em UTI, observamos que existe situações e decisões que exigem agilidade e responsabilidade, pois a UTI requer profissionais preparados para o enfrentamento de problemas éticos e interpessoais. Sendo assim a visita técnica como método de ensino-aprendizagem, permite vivenciar a experiência de estarem no futuro ambiente de trabalho através da observação das abordagens, rotinas empregadas, o cuidado, o contato direto com o paciente, a empatia e a postura do profissional diante das adversidades.

Portanto, a inserção nesse ambiente nos propiciou uma maior compreensão acerca da complexidade de uma UTI, e os profissionais que tivemos contato somaram de forma positiva em nossas vidas, compartilhando experiências vivenciadas dentro do setor, sendo inspiração através da empatia, do tratar igual e sem diferença. Essa visita técnica trouxe um aprendizado significativo, pois no decorrer da graduação, não nos deparamos com essa realidade de assistência de enfermagem prestada na UTI.

\section{DISCUSSÃO}

Entender o funcionamento da UTI é relevante para o aluno, sendo uma etapa fundamental para sua formação, haja vista que eles se deparam com um cenário diferente do seu cotidiano acadêmico, fazendo-os tomar atitudes de autonomia e liderança num setor de grande importância no ambiente hospitalar.

A academia provê aos alunos estágios em múltiplos campos, como também princípios e diretrizes básicas a serem adotados, cabendo ao aluno buscar o conhecimento e aperfeiçoar as técnicas, de acordo com as pretensões e objetivos a serem alcançados, incitando o pensamento crítico (NAZIAZENO SDS, et al., 2017; SOARES MI, et al., 2013).

Para Silva EFA, et al. (2013), a UTI começou da necessidade de conhecimento mas especializado acercar da assistência prestada a pacientes como doenças potencialmente grave, onde foram aprimorados e centralização de recursos materiais para atender de forma adequada clientes que se encontram em estado crítico, a partir da assistência multiprofissional especializada, contínua e ininterrupta. 
O enfermeiro tem um papel de grande importância dentro da UTI, pois ele é responsável por realizar: admissão do paciente no setor, passagem de plantão, a escala diária dos técnicos de enfermagem, evolução e registros de enfermagem de forma completa e legível, a fim de facilitar e organizar o trabalho da equipe, supervisionar e avaliar a assistência realizada pelos técnicos, além de prestar cuidados a pacientes que requerem maior complexidade e conhecimento técnico - cientifico que exigem a capacidade na tomada de decisões imediatas.

Também foi observado durante a visita, que o enfermeiro testa os equipamentos e aparelhos utilizados na UTI, atualiza o livro de registro do paciente, supervisiona e verifica os medicamentos do carrinho de emergência, através do check list, executam procedimentos como passagem de sondas, administração de medicamentos subcutâneos, curativos complexos, gasometria, entre outros. Evidencia-se, dessa forma, que os Enfermeiros são responsáveis pela gerência das unidades, atividade esta que prediz, providencia, realiza manutenção e o controle de recursos materiais e humanos para o bom funcionamento do serviço de saúde (OLIVEIRA NC e CHAVES LDP, 2009).

Na parte administrativa é responsabilidade do enfermeiro providência a realização de exames, prover recursos materiais, registrar pendência no livro de ocorrência, realiza notificações no Sistema de Nacional de Notificações para a Vigilância Sanitária (NOTIVISA) e no Software de Gestão de Riscos e Segurança do Paciente (VIGIHOSP), dentre outras funções. Concernente com o estudo de Pereira MCC, et al. (2019) que corroborou, dessa forma, a complexidade dos cuidados prestados pela equipe de enfermagem no ambiente da UTI, uma vez que esta envolve a presença de tecnologia sofisticada, exige profissionais habilitados e uma estrutura apropriada para uma assistência de qualidade.

Durante a visita foi possível constatar que os equipamentos são fundamentais para auxiliar os profissionais na prática do cuidar, uma vez que em casos de parada cardiorrespiratória, hipo ou hipertensão, choque e outros agravos, esses suportes são essenciais para alertar o atendimento precoce e prevenção de lesões irreversíveis ou até mesmo a óbito. A capacidade de manusear aparelhos eletrônicos e tecnologias empregadas como contribuintes no processo de recuperação do paciente também foi um fator citado pelo estudo de Massaroli R, et al. (2015) como algo que os motivava em relação às demais áreas assistenciais. Este ponto também contribui para a percepção de que dominar e desempenhar atividades entendidas como técnicas se sobrepõem até mesmo a premeditação do cuidado.

Os profissionais que nos acompanharam durante os dois dias de visita foram bastante receptivos, apresentando todo o funcionamento da parte técnica, clínica e a rotina de trabalho dentro da unidade, esclarecendo dúvidas, a respeito de procedimentos, admissões, equipamentos e cuidados prestados com pacientes internados. Com isto o acolhimento, segundo o Ministério da Saúde, pode ocorrer de várias maneiras, as quais incluem ações de saber ouvir e recepcionar indivíduos, presente invariavelmente nas relações de cuidado, que envolvem tantos trabalhadores como usuários (BRASIL, 2011). Para Pirolo SM, et al. (2011), a equipe de enfermagem é de grande importância dentro da Unidade de Terapia Intensiva, sendo um setor que apresenta situações complexa requer que os seus colaboradores sejam atentos e que estejam aptos a atender os pacientes em qualquer intercorrência de forma ágio e com a destreza necessária para realização de procedimentos.

O enfermeiro precisa ter uma visão global da unidade que gerencia, assim como o conhecimento do perfil dos pacientes, de si e de sua equipe. Necessita, igualmente, estar cauteloso às características individuais de cada um, de maneira a adaptar o tipo de atividade a ser desenvolvida. Recentemente, o enfermeiro, para enfrentar as transformações, carece criar novas formas de trabalho, perspectivas, novas ideias e objetivos, em busca de crescimento e de aperfeiçoamento, ampliação de habilidades, tornando-o capaz de realizar melhor suas funções, assistenciais e administrativas, com autonomia, informações essas repassadas pela enfermeira que nos acompanhou durante esta visita técnica. A visita técnica permitiu o entendimento organizacional do trabalho da enfermagem na UTI, revelando que o perfil de atuação dos profissionais que trabalham neste setor deve ser de iniciativa e atitude, além disso, ter o conhecimento técnico-científico e a capacidade de liderança é de suma importância, uma vez que prestam assistência a pacientes críticos que exigem cuidados complexos.

REAS/EJCH | Vol.Sup.n.53 | e3477 | DOI: https://doi.org/10.25248/reas.e3477.2020 Página 5 de 6 
Esta experiência, portanto, foi enriquecedora e essencial para a vida acadêmica das discentes, uma vez que foi o primeiro contato o setor, produzindo conhecimento e gerando novas experiências. $O$ acadêmico precisa conhecer os mais diversos setores que sua profissão lhe permite atuar e sabe-se que o principal campo de atuação da enfermagem no município onde moram abrange somente a assistência de baixa e média complexidade, o que torna esse estágio uma experiência imprescindível para a formação das futuras enfermeiras, contribuindo com sua qualificação profissional uma vez que o contato mais próximo com a nova realidade permite expandir os ainda mais seus horizontes.

\section{REFERÊNCIAS}

1. BRASIL MS. Secretaria de Atenção à Saúde. Departamento de Atenção Básica. Acolhimento à demanda espontânea. 56 p.: il. - (Série A. Normas e Manuais Técnicos) (Cadernos de Atenção Básica n. 28, Volume I). Brasília, 2011.

2. BRASIL MS. Secretária de Atenção á Saúde. Consulta Pública ํo 03, de 7 de julho de 2005. Diário Oficial da União, 2005 jul $08 ;$ no130.

3. BURGATTI JC, et al. Estratégias pedagógicas para o desenvolvimento da competência ético-política na formação inicial em Enfermagem/Pedagogical. Revista Brasileira de Enfermagem, 2013; 66(2): 1-282.

4. CASATE JC, CORREAA AK. Vivências de alunos de enfermagem em estágio hospitalar: subsídios para refletir sobre a humanização em saúde. Rev. Esc. Enferm. USP, 2006; 40(3):321-328.

5. DIAS EP, et al. Expectativas de alunos de enfermagem frente ao primeiro estágio em instituições de saúde. Rev.Psicopedagogia 2014; 31(94): 44-55.

6. FERNANDES HS, et al. Gestão em terapia intensiva: conceitos e inovações. Rev Bras Clin Med. São Paulo, 2011 mar-abr; 9 (2):129-37.

7. LEITE C, et al. Visita técnica em unidade de terapia intensiva como método de ensino-aprendizagem na graduação. Revista Eletrônica Acervo Saúde/Electronic Journal Collection Health- REAS/EJCH. Rio de Janeiro- RJ, 2019. $31: 1340$.

8. MASSAROLI R, et al. Trabalho de enfermagem em unidade de terapia intensiva e sua interface com a sistematização da assistência. Escola Anna Nery Revista de Enfermagem, Abr-Jun 2015. 19; (2):252-258.

9. MORTON PG, et al. Cuidados críticos de Enfermagem: Uma abordagem holística. 8. ed. Guanabara Koogan; Rio de Janeiro, 2007.

10. NAZIAZENO SDS, et al. Relato de experiência de acadêmicos de enfermagem frente ao estágio em unidade de terapia intensiva. Ciências Biológicas e de Saúde Unit. Aracaju, Out. 2017; 4(2):91-100.

11. OLIVEIRA NC, CHAVES LDP. Gerenciamento de Recursos Materiais: O Papel da enfermeira de Unidade De Terapia Intensiva. Rev. Rene. Fortaleza, out./dez.2009; 10(4): 19-27.

12. OUCHI JD, et al. O papel do enfermeiro na unidade de terapia intensiva diante de novas tecnologias em saúde. Revista Saúde em Foco, Edição ํo 10, 2018.

13. PEREIRA MCC, et al. Saberes e práticas do enfermeiro na unidade de terapia intensiva. Rev enferm UFPE online. Recife, 2019; 13(1):70-8.

14. PIROLO SM, et al. A integralidade do cuidado e ação comunicativa na prática interprofissional da terapia intensiva. Rev Esc Enferm. USP 2011; 45(6): 1396-1402.

15. SILVA EFA, et al. A humanização da equipe de enfermagem em unidades de terapia intensiva adulta. TCC (Graduação) - Curso de Bacharel Enfermagem, Faculdade Integrada de Pernambuco, Recife Pe, 2013.

16. SILVA GFD, et al. Refletindo sobre o cuidado de enfermagem em unidades de terapia intensiva. Revista Mineira de Enfermagem, 2007, 11(1):94-98.

17. SOARES MI, et al. Nursing process and its application in an intensive care unit: Integrative review. Rev enferm UFPE online, Recife, 2013; 7(5): 4183-91. 